\title{
Cryogenic Characteristics of the ATLAS Barrel Toroid Superconducting Magnet
}

\author{
R. Pengo, K. Barth, N. Delruelle, M. Pezzetti, O. Pirotte, G. Passardi, A. Dudarev, and H. ten Kate
}

\begin{abstract}
ATLAS, one of the experiments of the LHC accelerator under commissioning at CERN, is equipped with a large superconducting magnet the Barrel Toroid (BT) that has been tested at nominal current $(20500 \mathrm{~A})$. The BT is composed of eight race-track superconducting coils (each one weights about 45 tons) forming the biggest air core toroidal magnet ever built. By means of a large throughput centrifugal pump, a forced flow (about 10 liter/second at $4.5 \mathrm{~K}$ ) provides the indirect cooling of the coils in parallel. The paper describes the results of the measurements carried out on the complete cryogenic system assembled in the ATLAS cavern situated $100 \mathrm{~m}$ below the ground level. The measurements include, among other ones, the static heat loads, i.e., with no or constant current in the magnet, and the dynamic ones, since additional heat losses are produced, during the current ramp-up or slow dump, by eddy currents induced on the coil casing.
\end{abstract}

Index Terms-Cryogenics, eddy currents, fluid flow measurement, helium, superconducting magnets.

\section{INTRODUCTION}

$\mathbf{T}$ HE eight superconducting race-track coils forming the Barrel Toroid have been tested individually [1] on the surface at CERN, prior to their assembly in the ATLAS cavern. Once the BT system (about 360 tons of cold mass) was assembled in the cavern and then subsequently connected to the dedicated refrigerators in the ATLAS experimental area, measurements of the cooling down time, of the static heat load, and of the dynamic heat load were carried out. The behavior of the cryogenic system after a full current fast dump was also investigated.

\section{COOLING OF THE BARREL TOROID}

A detailed description of the ATLAS magnet cryogenic system can be found in [2], and the results of the first cooldown and operation at $4.5 \mathrm{~K}$ is described elsewhere [3] in this proceedings collection. With reference to Fig. 1 it is sufficient to recall here that a Shield Refrigerator (SR) is used for the pre-cooling and a Main Refrigerator (MR) is used in mixed liquefaction/refrigeration mode to maintain the level in the Phase Separator (PS) and in the Buffer Dewar (BD). Some $11 \mathrm{~g} / \mathrm{s}$ of Liquid Helium ( $\mathrm{LHe}$ ) are also used in pure liquefaction mode for the four pairs of Current Leads (CL). When the LHe

Manuscript received August 27, 2007.

R. Pengo, K. Barth, N. Delruelle, M. Pezzetti, O. Pirotte, and G. Passardi are with the Accelerator Technology Department, CERN, CH-1211 Geneva, Switzerland (e-mail: ruggero.pengo@cern.ch).

A. Dudarev and $\mathrm{H}$. ten Kate are with the Physics Department, CERN, CH-1211 Geneva, Switzerland.

Digital Object Identifier 10.1109/TASC.2008.921905

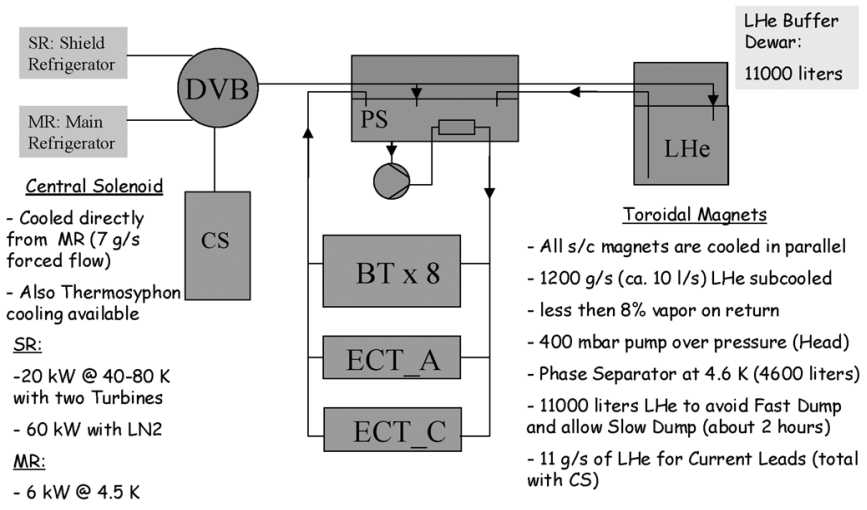

Fig. 1. A schematic view of the cryogenic ATLAS cooling system is shown The Shield Refrigerator (SR) is used for pre-cooling and for the thermal shields. The Main Refrigerator (MR) is connected, through a Distribution Valve Box (DVB), to the Phase Separator (PS) and to the Buffer Dewar (BD) to keep the Liquid Helium (LHe) level constant. The LHe centrifugal pump produces the sub-cooled LHe forced flow necessary to cool the Toroids.

level in PS, whose maximum capacity is 4600 liters, is higher than $70 \%$, one of the two high throughputs $(1200 \mathrm{~g} / \mathrm{s})$ liquid helium pumps [4] installed is started to produce the forced flow necessary to keep the magnets cold. The mass flow of the pumps, designed for the complete system, which includes the BT and the two End Cap Toroids (ECT), was "partialized" to obtain the nominal constant mass flow for each of the eight coils, and maintaining constant the pump rotational speed.

The result is obtained by diverting, directly in the PS, part of the flow generated by the pump. Due to the very high stability of the pump flow, each of the eight entrance valves of the BT coils are kept in a fixed position. Each cold mass is cooled by $80 \mathrm{~g} / \mathrm{s}$ of LHe forced flow.

\section{Static Heat LoAd of THE BARREl Toroid}

The measurements of the static heat load, both for the thermal shields and for the cold mass, of the complete system and of each individual coil were carried out. In addition, the total heat load of the eight thermal shields was measured to be $6000 \mathrm{~W}$, at the cooling mass flow of $67.6 \mathrm{~g} / \mathrm{s}$ and the outlet and inlet temperatures at $72.7 \mathrm{~K}$ and $56.4 \mathrm{~K}$ respectively.

In order to measure the individual static heat load of each coil, the rise of the average temperature of each cold mass was recorded, when the cooling of the $\mathrm{LHe}$ forced flow was stopped. The measurement was carried out keeping at the same time each thermal shield cooled by the SR at nominal values. From the known enthalpy values of each cold mass the static heat load was extracted.

The values can be found in Fig. 2, where they are also compared with those measured on the surface in the test station. A 


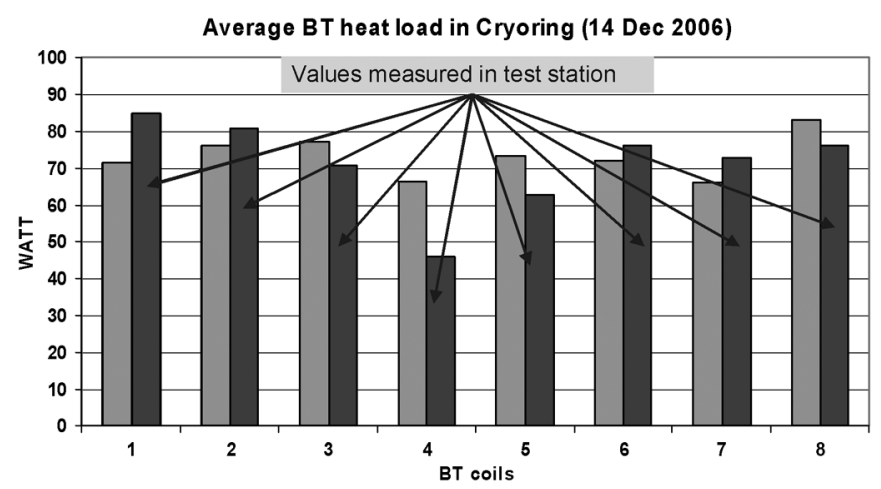

Fig. 2. Static heat load of each individual coil of the BT, both measured in the test station and in the cavern. The total heat load measured in the test final position is $586 \mathrm{~W}$, to be compared to the $571 \mathrm{~W}$ measured test station.

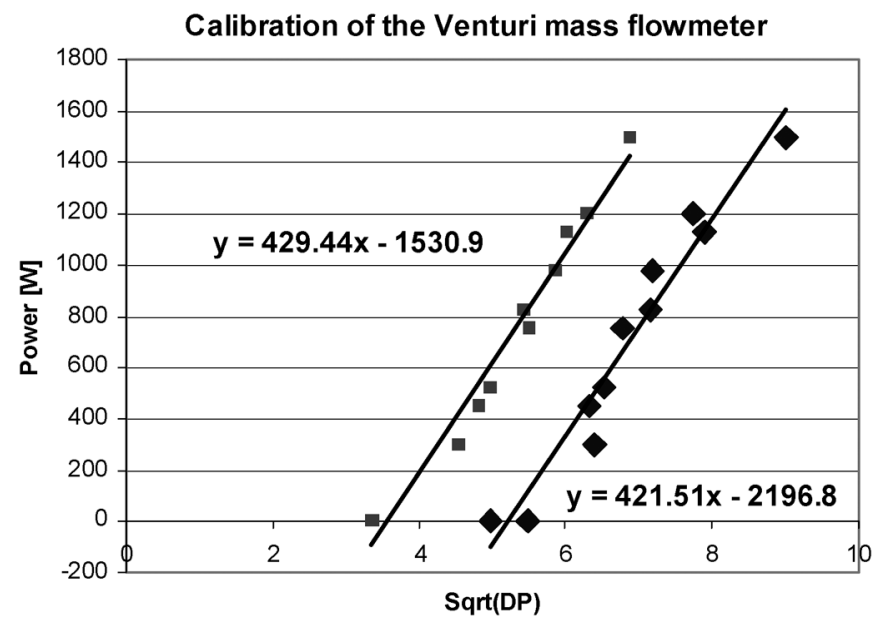

Fig. 3. The two curves show the calibration of the Venturi mass flow meter installed on the return line to the MR of PS. The left-hand side curve corresponds to the values obtained when withdrawing the LHe from the BD into the PS. The other curve is obtained when the PS is fed with LHe directly from the MR. The curves allow to extract the total heat load of the BT cryogenic system (see text).

very good agreement between the two measurements of the total heat load can be also appreciated in the summary graph.

The heat load measurement of the complete system was also carried out. The mass flow of the vapor returning to the refrigerator was measured with a Venturi mass flow meter calibrated by resistors immersed in the LHe of the PS. By varying the electric power dissipated by the resistors in LHe, the calibration of the Venturi can be extracted as well as the total heat load of the system. The measurement was done in two different ways.

At first (see the right-hand side of the graph in Fig. 3) the level in the PS was maintained directly by the MR. With this method the Venturi mass flow meter at the outlet of the PS measures both the vapor produced by the overall heat load plus the vapor produced by the long ( 80 meters) transfer line from the MR to the PS. The vapor due to the "flash" (vapor not condensed before leaving the MR) is also measured at the same time. The total result is about $2200 \mathrm{~W}$.

Moreover, the level in the PS was maintained constant (see left-hand side of the graph in Fig. 3) withdrawing the LHe from the 11000 liters BD, fed in turn by the MR. In this way the vapor

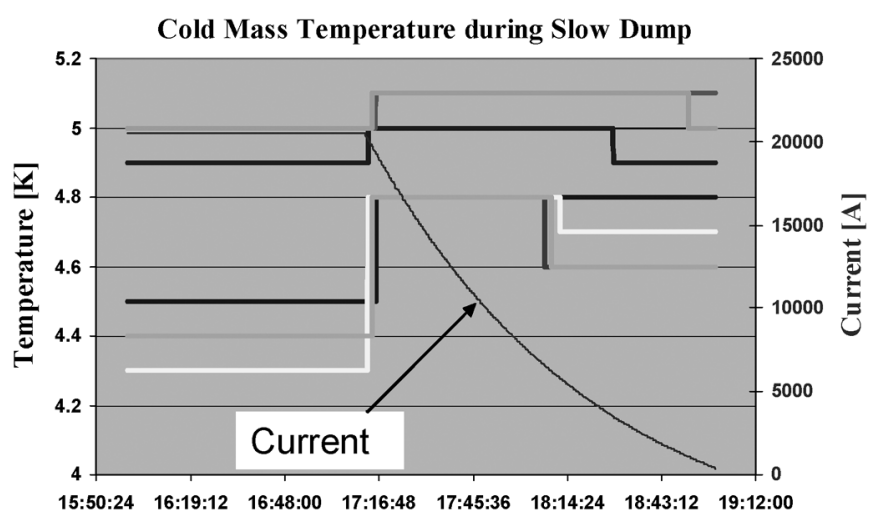

Fig. 4. Increase of temperature during slow dump. At first the slope of the change of current is higher causing the major increase, still less than $0.5 \mathrm{~K}$. The maximum temperature never exceeds the critical temperature of the superconductor at that current.

measured by the Venturi mass flow meter at the exit of the PS is corresponding to the heat load of the BT, the cryoring (the annular piping around the eight coils), the Proximity Cryogenic System (PCS), the LHe pumps and the transfer line (TL11) from the PCS to the cryoring. The total value measured is $1530 \mathrm{~W}$. The total heat load of the LHe pumps at the $1200 \mathrm{~g} / \mathrm{s}$ and 400 mbar of pressure head has been measured [4] to be $660 \mathrm{~W}$, which corresponds to a total efficiency of $60 \%$. The rest $870 \mathrm{~W}$ can be very reasonably split into $170 \mathrm{~W}$ for the cryoring (calculated value was $150 \mathrm{~W}$ ) plus transfer line TL11 and $100 \mathrm{~W}$ for the PCS (measured value), confirming the $600 \mathrm{~W}$ measured for the total BT heat load.

\section{DYNAMIC HEAT LOAD OF THE BARREL TOROID}

The coil casing is magnetically coupled to the BT (total inductance is $5.14 \mathrm{H}$ ), as a consequence currents are induced it when the current changes in 120 windings forming the coil [5].

The dynamic heat load was measured in the cavern during a slow dump discharge. When a slow discharge occurs the amount of additional heat load does not affect the normal cooling of the coils, i.e., the MR remains connected and the LHe pump continue to run without any perturbation.

Moreover the increase in temperature of the cold mass is less than $0.5 \mathrm{~K}$ (see Fig. 4) keeping the superconducting coils below their critical temperature.

For each coil the dynamic heat load has been measured on the surface test area at CERN with a constant di/dt during the ramp up of the current [1]. The results allowed to estimate that, for the BT, the total dynamic load at the average value of $4.2 \mathrm{~A} / \mathrm{s}$ would be around $350 \mathrm{~W}$. The slow dump of the ATLAS BT is not evolving with a constant slope di/dt during the two hours necessary to discharge completely the magnet from $20500 \mathrm{~A}$.

In facts, the total electromagnetic energy (EM), about $1 \mathrm{GJ}$ at $20.5 \mathrm{kA}$, is dissipated onto a combination of diodes and resistors, resulting on a roughly exponential decay. As a result of the additional heat load on the cold mass, more LHe is vaporized.

The vapor can be detected as an increase of the helium vapor exiting the PS. An average of $380 \mathrm{~W}$ was measured by means of the Venturi mass flow meter (see Fig. 5 installed on the return line from the PS to the MR). The measurement is not highly 


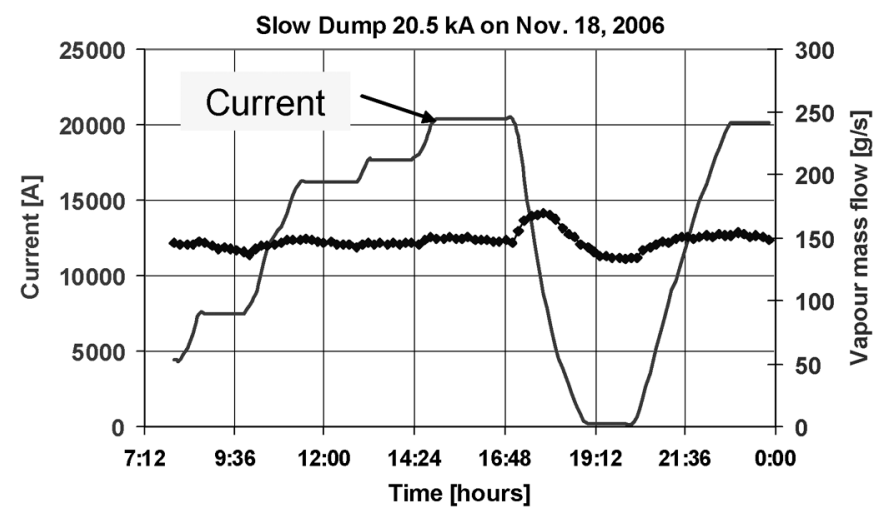

Fig. 5. Dynamic heat load as measured by the Venturi mass flow meter on the outlet of the Phase Separator towards the Main Refrigerator. The mass flow increase corresponds to an average heat load of $380 \mathrm{~W}$.

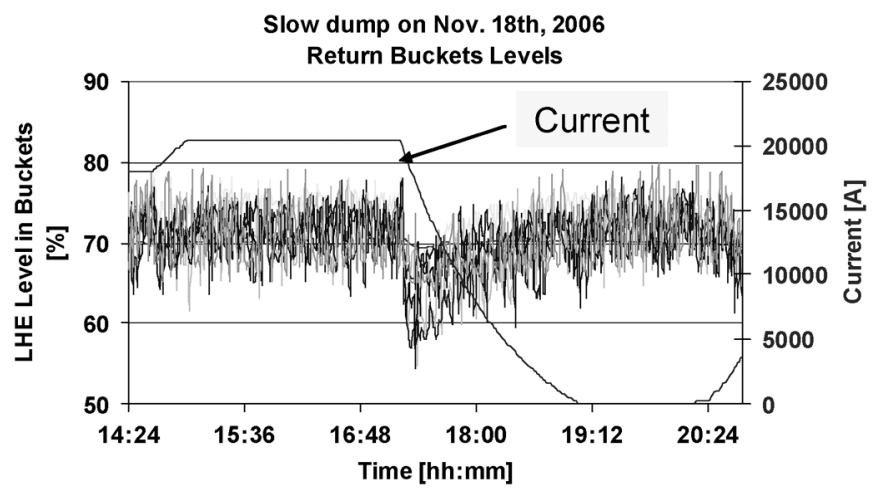

Fig. 6. Liquid helium level in each of the buckets on the return line of a BT coil is shown during a slow dump. Due to dynamic heat load the amount of vapor increases and the LHe level in the return buckets decreases.

precise due to the dynamic changing of the pressure in the PS, and an accuracy of about $10 \%$ is estimated.

Moreover, each of the buckets with a hole, which are installed in the PS at the end of the return line from each coil, shows clearly, during a slow dump, a decrease of the LHe level. In Fig. 6 the behavior of the LHe level in the buckets is shown, together with the slow decrease of current in the magnets. The heat load is proportional to the $\mathrm{di} / \mathrm{dt}$, squared, which produces the shape of the LHe level in the buckets: at first the di/dt is very high then it decreases. Knowing the mass flow entering each coil, which is measured at its entrance before the relevant valve, the buckets level variation can be used to evaluate the quantity of vapor (i.e., as quality meters) produced in the coil, which in turn allows to extract the additional heat load. The peak value corresponds to about $100 \mathrm{~W}$ per coil, whilst the average is half, corresponding to a total average of $400 \mathrm{~W}$ for the eight coils, in good agreement with the value $(380 \mathrm{~W})$ measured with the Venturi mass flow meter.

\section{CRYogenics of the Fast Dump of the Barrel Toroid}

The BT was also discharged abruptly by a Fast Dump (FD) when charged at different values of the current, and in particular at full $(20500 \mathrm{~A})$ nominal current. During the few minutes that a FD takes, the complete EM energy stored (about $1 \mathrm{GJ}$ ) is mainly dissipated in the cold mass.
Fast Dump Temperature

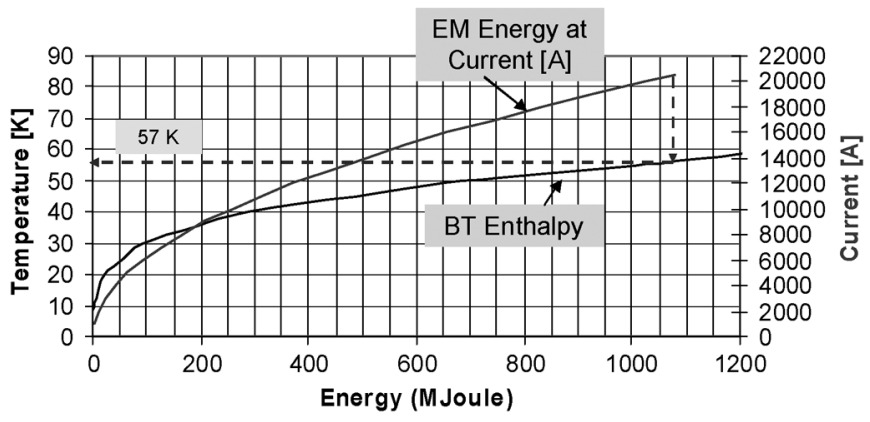

Fig. 7. The graph shows an easy way to calculate the maximum temperature that the BT cold mass reaches as a consequence of a Fast Dump (FD). On the $\mathrm{x}$-axis the energy is displayed, given by the enthalpy of the BT at a given temperature (on left-hand side) or by the current (right-hand side). If all EM energy is released into the cold mass its temperature after a FD should be $57 \mathrm{~K}$.

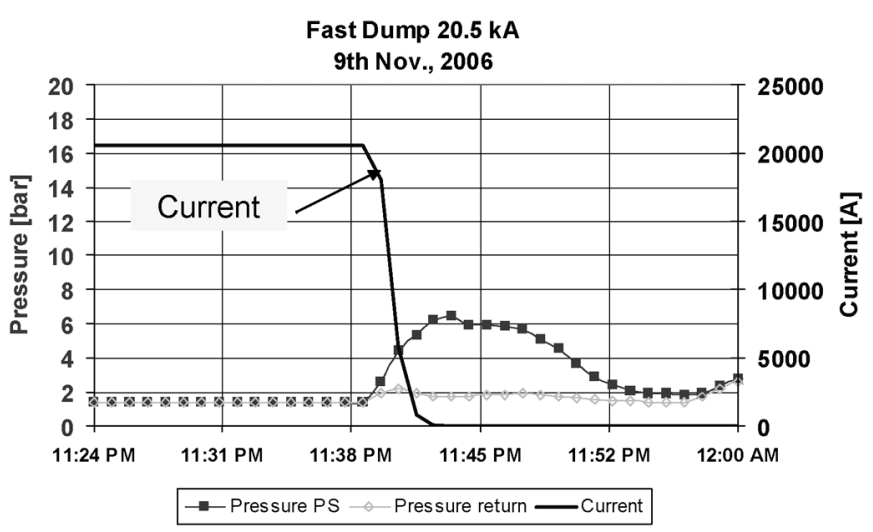

Fig. 8. Pressure rise as a consequence of the Fast Dump: the value in the Phase Separator is kept around 6 bars (intermediate curve). The value at the bottom corresponds to the pressure on the return line of the SR compressor. No helium was released into the atmosphere since the opening set point of the pressure relief valves is 21 bars.

The current flows then in the Aluminum casing as a consequence of the firing of the quench heaters, which uniformly propagate the transition above the critical temperature of the superconductor. The result is an increase of the cold mass temperature up to $58 \mathrm{~K}$.

In Fig. 7 a graphical easy way to predict the maximum temperature of the cold mass is shown.

A digital signal, sent to the cryogenic control system, when the FD is initiated, disconnects the MR from the system and stops the LHe pump running. The SR remains connected continuing the cooling of the thermal shields. The vapor produced by the high temperature rapidly reached by the cold mass $(58 \mathrm{~K}$ measured) is sent to the PS and from there, in a controlled manner, to the suction of the SR compressor on the surface. The pressure in the PS is then kept at a reasonable value which never overcame the 6 bar absolute $(600 \mathrm{kPa})$ at $20500 \mathrm{~A}$. It has to be noted that the aperture set point of the pressure relief valves of the different cryogenic components is $21 \mathrm{bar}(2.1 \mathrm{MPa})$ gauge, well above the maximum pressure produced. No helium was released into the atmosphere as a consequence of a FD. In Fig. 8 the helium pressure rise in the PS is shown as a function of time from the start of the FD. 


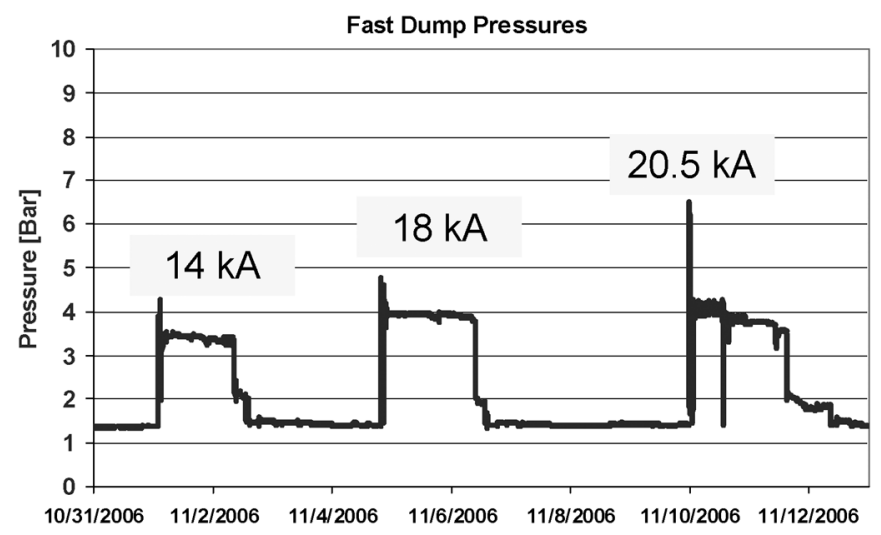

Fig. 9. In the graph the different values of the maximum pressures in the Phase Separator (PS), which correspond to the Fast Dumps (FD) at the relevant current.

In Fig. 9 the different pressure values reached at different value of the current at which the FD occurred. It is clearly visible that at a higher current value corresponds a higher maximum pressure in the PS.

As soon as the pressure in the PS is back at its nominal value below 1.4 bars and no LHe is present, the re-cooling of the BT is started [3].

\section{Pump Switching at Full CurRent}

Only one of the two LHe centrifugal pumps installed is running, the second one being redundant and ready for use without warming up the pump cryostat. The complete PCS is connected to a battery system (UPS), which allows the LHe pumps operation also in case of a main power failure. The electric energy stored in the UPS, as well as the amount of helium in the BD, are such that they allow a slow discharge of the magnet from full current, during the two hours needed. The switching from one pump to the redundant one takes about 40 seconds, the time necessary to speed up the pump from zero to $76 \mathrm{~Hz}$. During that time the cold mass is kept at nominal temperature without any problem, whilst the LHe at the bottom of each Current Lead is not sufficient to maintain the $1.7 \mathrm{~g} / \mathrm{s}$ required mass flow and, when a failure was simulated to the running pump, the protection system triggered a Fast Dump. Tests are planned, at CERN pump test station, to investigate if both LHe pumps could run simultaneously.

\section{CONCLUSIONS}

The different measurements carried out on the BT system have been described: they include the static heat load measured with two different methods, the dynamic heat load at full current discharge, and the cryogenics of the fast dump from full current. The values were also compared to those taken on the surface for each individual coil forming the BT, and found to be in very good agreement.

\section{ACKNOWLEDGMENT}

The authors thank R. Baud, D. Legrand, O. Pavlov for their constant assistance for the control system during the measurements.

\section{REFERENCES}

[1] K. Barth, N. Delruelle, A. Dudarev, S. Junker, R. Pengo, O. Pirotte, and C. Berriaud, "Results of the cryogenic test of the superconducting magnets forming the barrel toroid of the ATLAS experiment," Advances in Cryogenic Engineering, vol. 51B, pp. 1628-1634, 2006.

[2] N. Delruelle, F. Haug, H. ten Kate, G. Passardi, R. Pengo, M. Pezzetti, O. Pirotte, E. Baynham, T. Bradshaw, and R. Rochford, "Commissioning of the cryogenic system for the ATLAS superconducting magnets," Advances in Cryogenic Engineering, vol. 51B, pp. 2018-2025, 2002.

[3] K. Barth, A. Dudarev, F. Haug, N. Delruelle, G. Passardi, R. Pengo, M. Pezzetti, O. Pirotte, and H. Ten Kate, "First cool-down and test at 4.5 $\mathrm{K}$ of the ATLAS superconducting barrel toroid assembled in the LHC experimental cavern," IEEE Trans. Appl. Superconduct., vol. 18, no. 2, Jun. 2008.

[4] R. Pengo, S. Junker, G. Passardi, O. Pirotte, and H. ten Kate, "Test results of a $1.2 \mathrm{~kg} / \mathrm{s}$ centrifugal liquid helium pump for the ATLAS superconducting toroid system," in Proceedings of the 19th International Cryogenic Engineering Conference, G. Gistau Baguer and P. Seyfert, Eds., Grenoble, France, 2002, pp. 71-74.

[5] R. Pengo, N. Dolgetta, S. Junker, G. Passardi, and H. ten Kate, "Heat load measurements on a large superconducting magnet: An application of a void fraction meter," IEEE Trans. Applied Superconductivity, vol. 16, pp. 1704-1707, 2004. 